\title{
Low-Cost Fluorescent Microvascular Visualization in Ambystoma mexicanum
}

\author{
Luke Bollinger and Renee Dickie
}

Towson University, Towson, Maryland, United States

Blood vessel imaging allows researchers to explore the role of the vasculature in physiology, pathogenesis, tissue regeneration, and more. This study introduces a low-cost method for fluorescent visualization of the microvasculature in Ambystoma mexicanum, the premier animal model system for vertebrate regeneration. Successful visualization of microvasculature during regeneration is essential for understanding the role of angiogenesis in tissue regrowth and repair. Here, we modify and extend methods developed for chick and mouse embryos and zebrafish (Takese et al. 2013; van Gennip et al., 2018) to visualize vessels in salamanders, using highlighter ink as a fluorescent label and imaging using a fluorescent adapter system with a stereomicroscope and phone camera.

Methods

Animals were obtained from the Ambystoma Genetic Stock Center (Lexington, Kentucky). Axolotls were individually housed in tanks containing 40\% Holtfreter's solution and fed a diet of Rangen pellets. All work was conducted in accordance with an approved Towson University Institute for Animal Care and Use Committee protocol (1611000157R001). Two axolotl strains, white and wildtype, were compared for their suitability for the protocol. The white strain (AGSC_101E) proved superior as it as lacks skin pigmentation that can obscure the vascular label.

Vascular perfusion with fluorescent marker

Fluorescent yellow highlighter ink (Pilot Spotliter Supreme, Pilot Corporation, Jacksonville FL) was diluted 1:1500 in $40 \%$ Holtfreter's solution prior to use. For adult animals, $4.5 \mathrm{ml}$ of solution was drawn into a $5 \mathrm{ml}$ syringe with a 26.5 gauge needle. Cardiac and aortic perfusion were performed as described in Montoro \& Dickie (2017). Perfused tissue was collected from euthanized animals and fixed in paraformaldehyde.

Fluorescent Detection

Fluorescent images of the tail were obtained using a Bausch \& Lomb stereomicroscope with a Celestron digital camera accessory and a NIGHTSEA Model SFA Stereo Microscope Fluorescence Adapter (NIGHTSEA, Lexington MA). Images were captured using Micam software. A second set of images was obtained using an iPhone 11 12-megapixel TrueDepth camera with the filter placed over the lens. This allowed for visualization of the entire tail in a single frame. Exposure was adjusted over the entire field in one of the images (Fig. 1E) to limit background interference. In some cases, image background was cropped for formatting into figures; no other image postprocessing manipulations were performed.

Results and Discussion

Despite serving as a model system for vertebrate wound repair and regeneration, there are relatively few available techniques with which to explore the microvasculature in axolotls. The techniques described here provide a method to label the microvasculature, including capillary networks (Fig. 1), in this amphibian and capture the necessary low magnification large fields of view of the tissues of interest. The fluorescence was detectable both imaging by stereomicroscope (Fig. 1A-D) and with a cell phone camera (Fig. 1E-F), which allowed for capture of larger fields of view. Much of the fluorescent signal survived paraformaldehyde fixation (Fig. 2). On occasion, there were some punctate aggregates of the ink (Figure 1C, circles), which we expect could be eliminated with further dilution, sonication, or vortexing of the solution. In some areas, 
perfusion with the fluorescent label was not complete (Fig.1D, arrowhead). The administration of heparin prior to perfusion in subsequent experiments may alleviate this.

The advantages of this vascular labeling technique are its accessibility, simplicity, and low cost. In contrast to other fluorescent agents conventionally used for vascular perfusion (e.g., fluorescently labeled lectins or dextrans), the fluorescent ink is inexpensive and widely available as an office supply. The ink has been demonstrated to specifically label endothelial cells in avian and mouse embryos and the signal persists through fixation and immunohistochemistry (Takase et al., 2013; Takahashi et al., 2015), making it useful for dual labeling experiments. The imaging set up, employing the NIGHTSEA adapter with a dissecting scope or cell phone was also very cost effective compared to standard fluorescent microscopy. We focused on recording images of tail microvasculature, as our work centers on the regeneration of this appendage, however the technique is applicable to all the vasculature through out the animal, and could be applied to other salamander species as well. This technique adds to the set of tools for observing microvasculature in urodeles, and given its low cost and accessibility, is particularly well-suited for classroom-based research inquiry by students interested in the role of blood vessels in tissue repair and regeneration.

\section{Acknowledgements}

Animals were obtained from the Ambystoma Genetic Stock Center, which is supported by NIH P40OD019794.
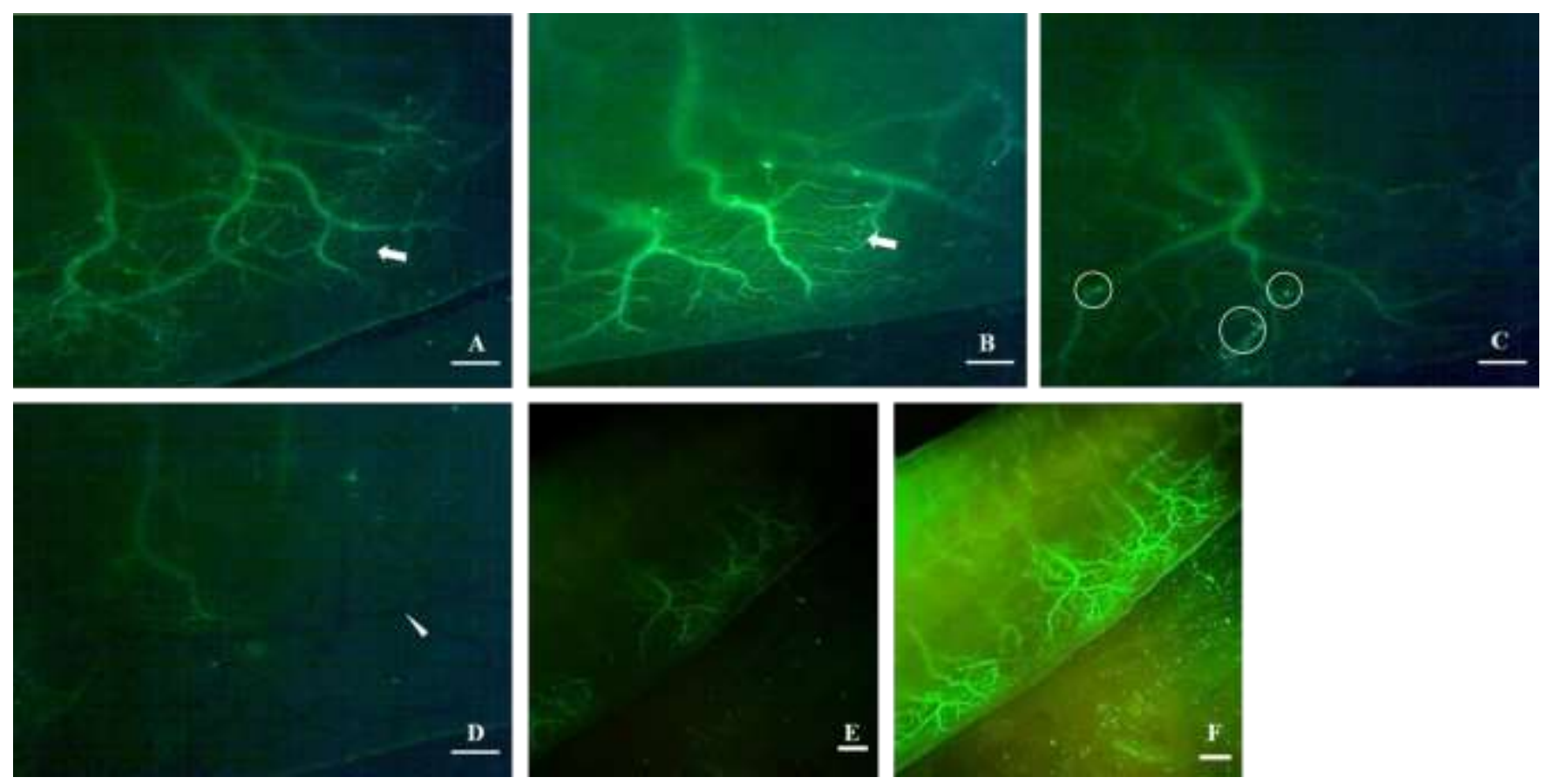

Figure 1. Microvasculature can be visualized in multiple locations in the tail following ink perfusion and imaging with a stereomicroscope (A-D) or phone (E-F). Fluorescent signal from the ink is visible in capillary networks (A \& B, solid arrows) as well as larger vessels. Some areas appeared to contain aggregates of fluorescent signal (C, circles). The proximal tail was well perfused, while more distally, in some areas, perfusion was incomplete ( $\mathrm{D}$, pointer). Tail microvasculature was also imaged using an iPhone 12MP TrueDepth Camera with reduced (E) and full exposure (F). Scalebars $=1 \mathrm{~mm}$. 


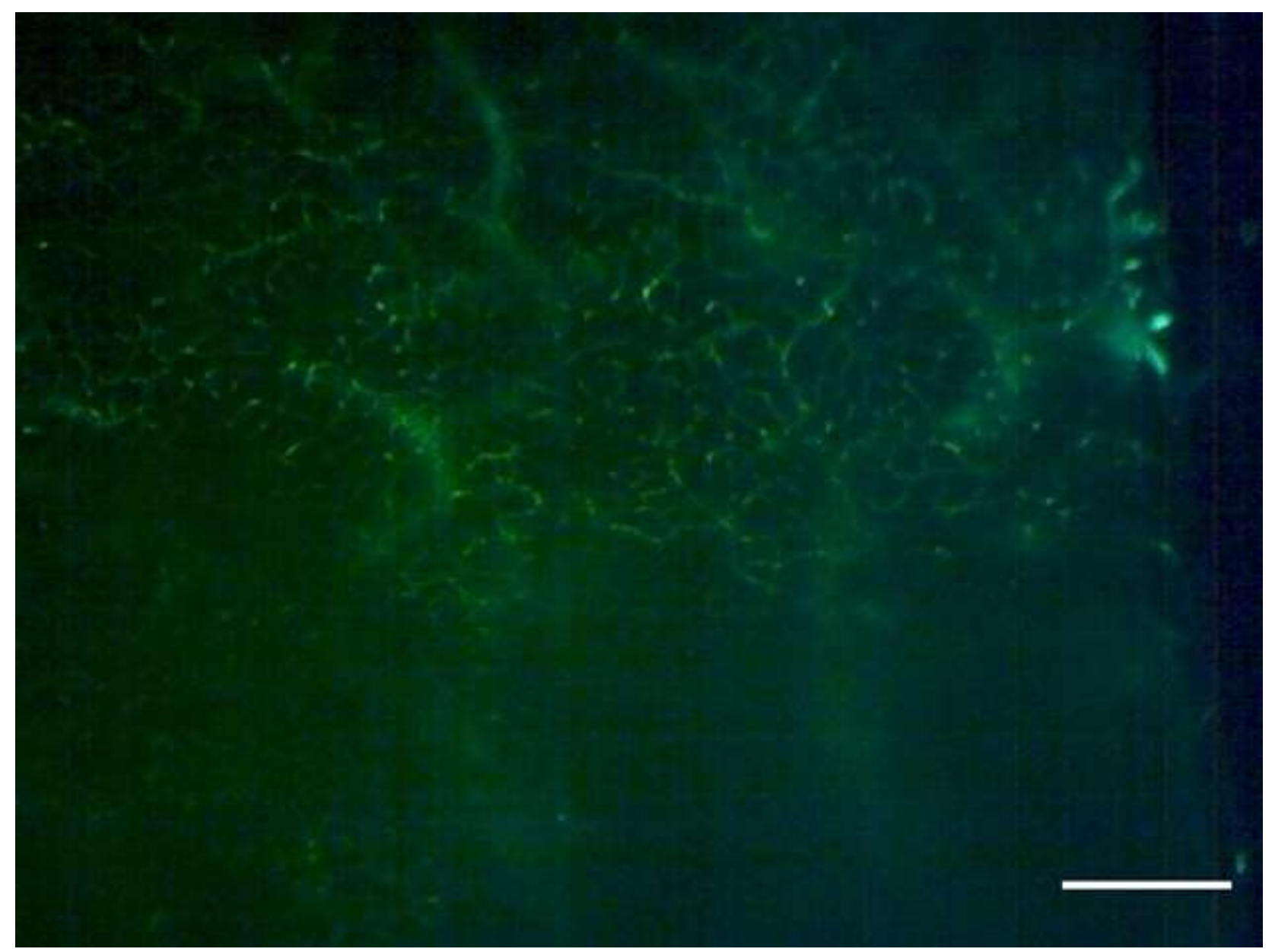

Figure 2. Vessels following 6 days of paraformaldehyde fixation. Scale bar=1mm.

\section{References}

MONTORO, R. \& DICKIE, R. (2017). Comparison of tissue processing methods for microvascular visualization in axolotls. MethodsX 4:265-273

TAKAHASHI, T., TAKASE, Y., YOSHINO, T., SAITO, D., TADOKORO, R. \& TAKAHASHI, Y. (2015). Angiogenesis in the developing spinal cord: Blood vessel exclusion from neural progenitor region is mediated by VEGF and its antagonists. PLoS ONE 10(1):e0116119

TAKASE, Y., TADOKORO, R. \& TAKAHASHI, Y. (2013). Low cost labeling with highlighter ink efficiently visualizes developing blood vessels in avian and mouse embryos. Development Growth and Differentiation 55(9):792-801

VAN GENNIP, J. L. M., BOSWELL, C. W. \& CIRUNA, B. (2018). Neuroinflammatory signals drive spinal curve formation in zebrafish models of idiopathic scoliosis. Science Advances 4(12): eaav1781 